\title{
Impact des activités non agricoles sur la sécurité alimentaire au Sud-Kivu montagneux
}

Angélique Neema Ciza, Clérisse Casinga Mubasi, Richard Amani Barhumana, Delvaux Kabike

Balyahamwabo, Jean-Luc Namegabe Mastaki \& Philippe Lebailly

Angélique Neema Ciza : Doctorante en Economie et Développement rural, Assistante de recherche et d'enseignement, Faculté des Sciences économiques et de gestion, Université Evangélique en Afrique, Congolaise, Bukavu, Sud-Kivu, République démocratique du Congo. Auteur correspondant : E-mail : Angelique.NeemaCiza@student.uliege.be / neema_ciza@yahoo.fr GSM : +32471872408

Clérisse Casinga Mubasi : Doctorant en Sciences agronomiques, Cassava Disease Specialist, Congolais, Institut International d'Agriculture Tropicale (IITA), Bukavu, République démocratique du Congo. E-mail : C.Casinga@cgiar.org

Richard Amani Barhumana : Licencié en Economie Rurale, Université Evangélique en Afrique, Congolais, Bukavu, Sud-Kivu, République démocratique du Congo. E-mail : amanibarhu@gmail.com

Delvaux Kabike Balyahamwabo : Msc student in organizational Psychology (Unicaf), Administrative Officer, Congolais, Institut International d’Agriculture Tropical (IITA), Bukavu, SudKivu, République démocratique du Congo. E-mail : B.Kabike@cgiar.org

Jean-Luc Namegabe Mastaki : Docteur en Sciences agronomiques, Senior Economist and Team Leader Raw Materials, Horticulture and Tropical Products (RAMHOT), Trade and Markets Division (EST), Congolais, FAO, Rome, Italie. E-mail : lekimastaki@yahoo.fr

Philippe Lebailly : Docteur en Sciences agronomiques, Professeur et responsable du Laboratoire d’Economie et Développement rural de Gembloux Agro-Bio Tech, Université de Liège, Belge, Passage des Déportés, 2, 5030 Gembloux, Belgique. E-mail : philippe.lebailly@uliege.be

DOI: $10.25518 / 2295-8010.1761$

\section{Résumé :}

Selon les résultats de l'analyse participative de la pauvreté (28), la crise économique et financière actuelle, qui s'étend désormais à tous les pays sous-développés et en développement, affecte gravement l'économie de la République démocratique du Congo et, plus particulièrement, les ménages ruraux de la province du Sud-Kivu, qui sont touchés par des crises récurrentes sans précédent et qui les plongent dans une pauvreté sévère et insoutenable. Paradoxalement, ces agriculteurs, dont le rôle est de fournir une nourriture saine et abondante à la population congolaise, sont parmi les moins bien nourris. L'objectif de cette étude est de diagnostiquer l'impact de l'activité non-agricole sur la sécurité alimentaire des ménages du SudKivu montagneux dans la collectivité-chefferie de Luhwindja. Les résultats révèlent que 37\% des ménages ruraux considérés tirent une partie de leurs revenus des activités non agricoles, ce qui améliore leur sécurité alimentaire, tandis que 63\% des ménages n'ayant que l'agriculture comme 
Impact des activités non agricoles sur la sécurité alimentaire au Sud-Kivu mo...

moyen de subsistance ont un niveau de sécurité alimentaire critique car ils ne sont pas en mesure d'obtenir 2400 kilocalories par jour et par personne. Le secteur non agricole et les revenus des activités non agricoles sont donc très importants et contribuent de manière significative à l'économie des ménages ruraux en raison de leur influence sur le niveau de vie des ménages et la sécurité alimentaire de ceux-ci.

\section{Abstract :}

\section{Impact of non-agricultural activities on food security in mountainous South Kivu.}

According to the results of the participatory poverty analysis (28), the current economic and financial crisis, which is now extending to all underdeveloped and developing countries, is severely affecting the economy of the Democratic Republic of the Congo and, more specifically, rural households in South Kivu province, which are affected by unprecedented recurring crises that plunge them into severe and unsustainable poverty. Paradoxically, these farmers, whose role is to provide healthy and abundant food to the Congolese population, are among the least wellfed. The objective of this study is to diagnose the impact of non-agricultural activity on household food security in mountainous South Kivu in the Luhwindja chieftaincy community. The results revealed that $37 \%$ of the rural households considered derive part of their income from non-agricultural activities, which improves their food security, while $63 \%$ of households with only agriculture as a means of subsistence had a critical level of food security because they were unable to obtain 2400 kilocalories per day per person. The non-agricultural sector and income from non-farming activities are therefore very important and contribute significantly to the economy of rural households because of their influence on the standard of living of households and their food security.

\section{Introduction}

La crise économique et financière de 2008 qui a suivi la crise alimentaire de 2007 et qui a touché de plein fouet l'ensemble des pays les moins avancés et en développement, a très durablement affecté l'économie de la République démocratique du Congo et plus particulièrement celle de la province du Sud-Kivu $(24,12)$. Cette situation a entraîne une baisse de la production agricole conduisant à une aggravation de la situation alimentaire de telle sorte qu'environ 70 pour cent de la population n'a pas accès à une nourriture suffisante, (25).

Le Sud-Kivu est confronté à plusieurs défis pour assurer la sécurité alimentaire de sa population, notamment la dépendance des pluies pour la réussite de la campagne agricole, la dégradation des sols $(9,10,3)$, le faible accès aux services sociaux de base, une pauvreté parfois stagnante, la stabilité politique ainsi que l'inexistence d'une politique agricole cohérente en phase avec un exode rural corollaire d'explosion démographique comme observé ailleurs en Afrique (17).

Dans les villages, la vision traditionnelle veut que les ménages agricoles se consacrent de façon quasi exclusive à l'agriculture et n'entreprennent que de façon marginale des activités rurales non agricoles, pourtant en évolution croissante $(15,25)$. Ainsi, le revenu non agricole occupe une place de plus en plus importante dans l'économie rurale et la sécurité alimentaire dans les pays en développement $(6,25)$. De nombreux auteurs témoignent de la nécessité de soutenir l'emploi non agricole tant en milieu urbain que rural.

Dans les pays en développement, des études ont mis en évidence que le pourcentage de la population 
rurale employée de façon permanente dans les activités rurales non agricoles variait entre $14 \%$ et 19\% (Chuta \& Liedlom, 1979 ; Hagglade et al., 1987, cités par (4). En aval, l'agriculture constitue une source d'emploi pour 30 à 50\% de la population (Chuta et Liedlohm, 1979, cités par (4), elle améliore la situation économique des ménages agricoles et accroît ainsi le revenu rural $(19,34$, 37). Les activités non agricoles constituent une composante quantitative importante de l'économie locale pour les ménages dans les campagnes $(13,22)$.

Dans le milieu rural, et plus particulièrement dans ceux du Sud-Kivu, elles constituent une réalité mal connue et pas ou peu documentée, ni chiffrée tant par les techniciens, les administrations locales, nationales et internationales que par les premiers intéressés eux-mêmes (16). La plupart des recherches démontrent l'écart considérable entre le revenu des activités agricoles et extraagricoles (1).

L'amélioration dans l'ensemble du revenu rural, la participation aux activités non agricoles rurales pourraient réduire les disparités de cette source de revenu, surtout dans les zones pauvres $(5,26$, 32) car la génération de revenu des ménages ruraux provient de la force de travail des personnes engagées dans les emplois divers et de la vente des biens et services produits (22). En outre, au fur et à mesure que la part du revenu non agricole augmente dans le revenu total du ménage, la distribution de ce dernier devient plus uniforme et permet aux ménages de répondre d'une manière satisfaisante aux besoins du ménage face à l'insécurité alimentaire (1) et ainsi développer certaines stratégies de résilience (14).

L'objectif de la présente étude est d'analyser l'impact de l'activité non agricole sur la sécurité alimentaire des ménages du Sud-Kivu montagneux dans la collectivité-chefferie de Luhwindja tributaire de plusieurs guerres successives.

\section{Méthodologie}

\section{Délimitation géospatiale}

Couvrant une superficie de $11007 \mathrm{~km}^{2}$ à l'est de la province du Sud-Kivu, le territoire de Mwenga est composé de la collectivité-chefferie de Luhwinja qui est une des six chefferies que compte le territoire avec celles de Basile, de Burhinyi, d’Itombwe, de Luindi et de Wamuzima. La collectivitéchefferie de Luhwinja étant à son tour composée de 9 groupements dont Bujiri, Kabalole, Karhundu, Mulama, Luciga, Luduha, Cibanda, Idudwe et Burhembo. Elle est traversée d'Est en Ouest par la rivière Namunana et renferme plusieurs gisements miniers exploités tant de manière industrielle qu'artisanale (29). 


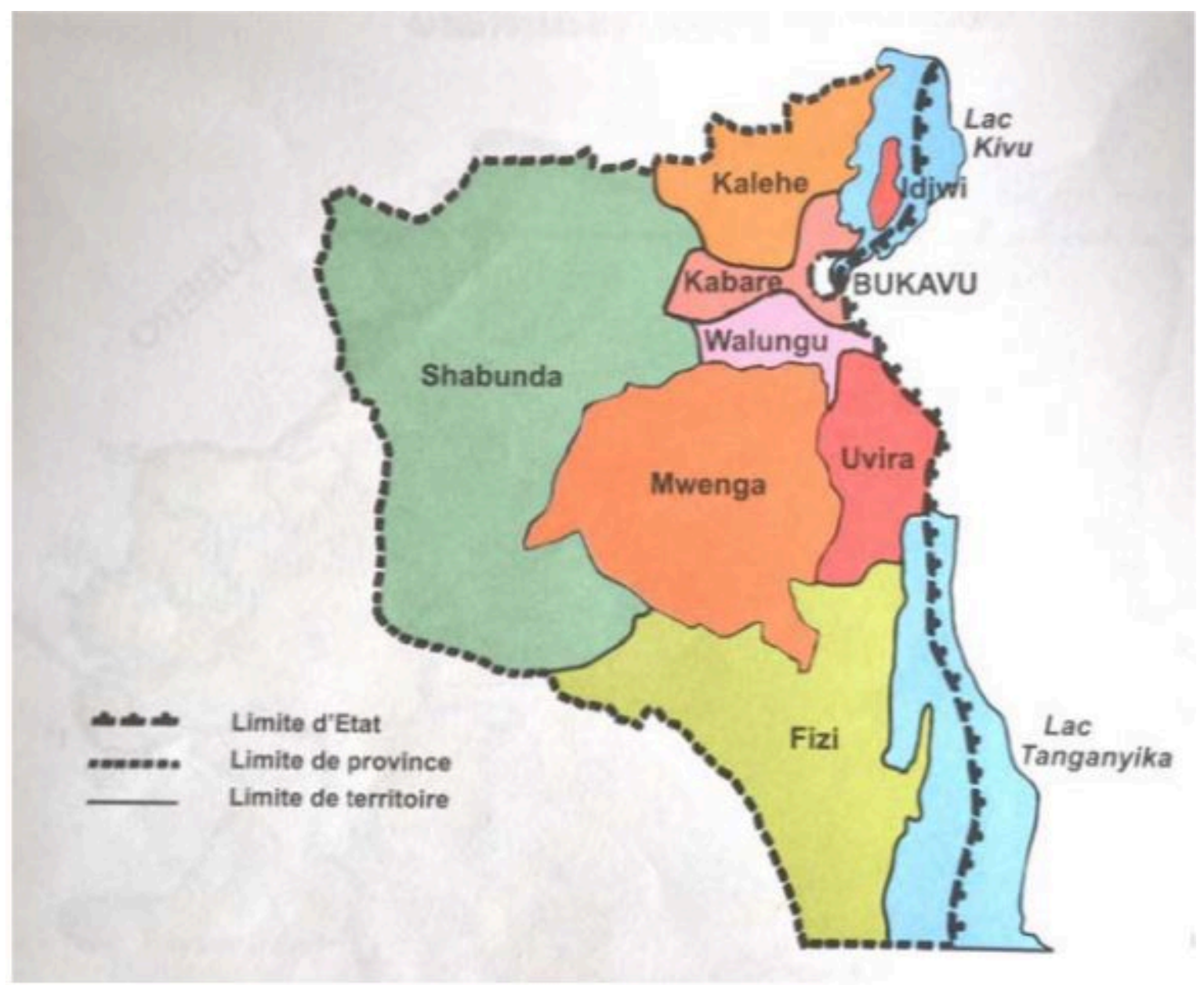

Carte 1. Le territoire de Mwenga dans la province du Sud-Kivu

Source : De Saint Moulin L. \& Kalombo Tshibanda J.-L., 2005 (11) 


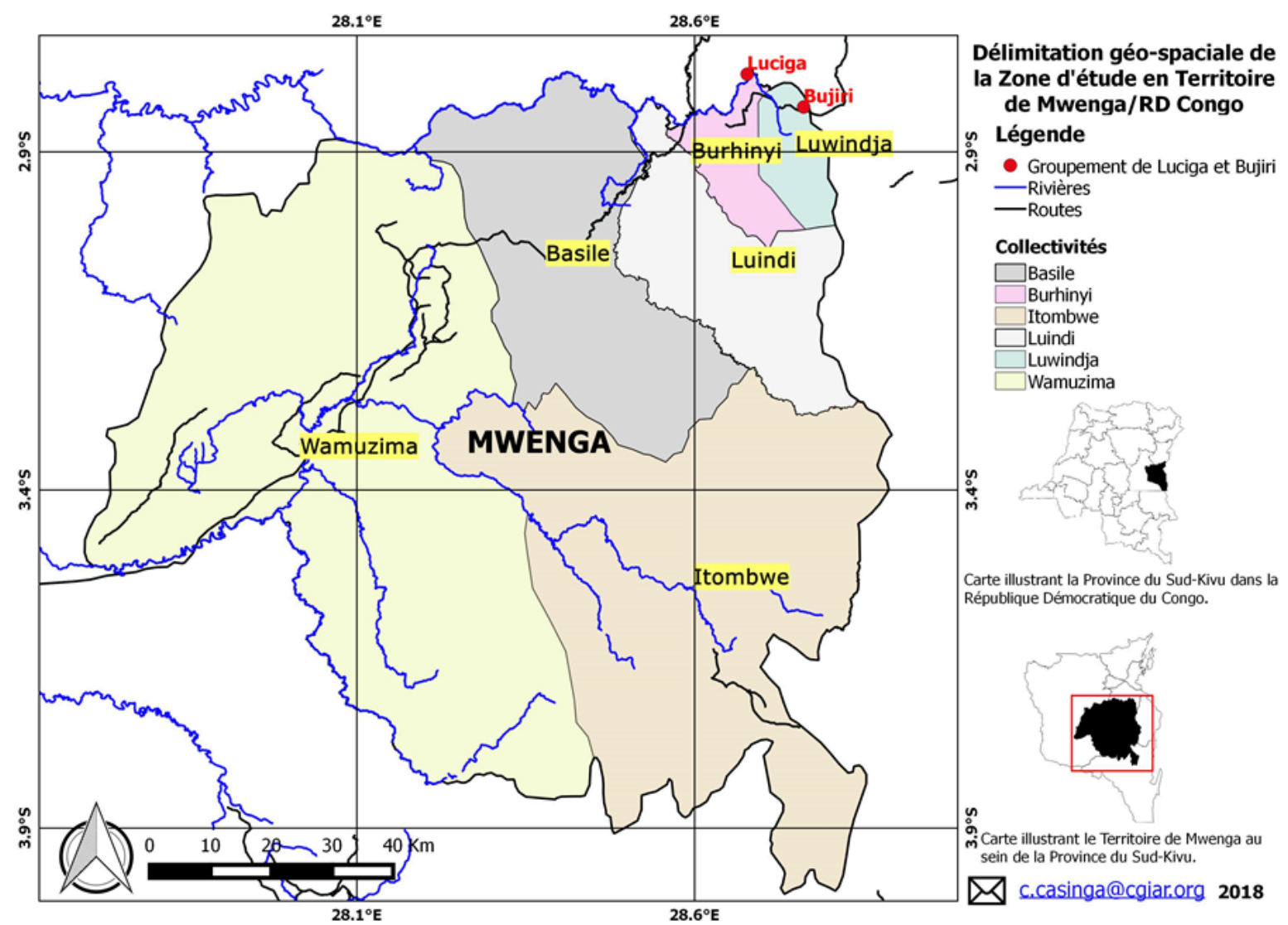

Carte 2. Carte administrative du territoire de Mwenga

\section{Méthodes et techniques}

Dans la présente étude, l'enquête à un seul degré est la technique correspondante où la population cible est celle des ménages ruraux de deux groupements, à savoir ceux de Bujiri et de Luciga. L'enquête a été menée au cours de l’année 2015-2016. Une pré-enquête réalisée au cours du mois de novembre 2015 a permis de déterminer la taille de l'échantillon sur laquelle a porté cette étude. Ensuite, le questionnaire a été testé auprès d'une sélection d'individus de la population concernée par l'étude.

Cette pré-enquête a considéré la variable d'intérêt qui était la pratique ou non des activités non agricoles et la part de ces activités dans le revenu des ménages avec comme réponses attendues OUI ou NON, significative ou non significative. Ainsi, les ménages étaient enquêtés de façon aléatoire avec un pas d'échantillonnage de 3 ménages, en considérant les deux zones ayant fait l'objet de cette étude.

Dans le groupement de Bujiri, sur les 30 ménages pré-enquêtés, 7 (23\% des enquêtés) ont répondu OUI, donc pratiquant des activités non agricoles et 23 enquêtés (77\% des enquêtés) ont répondus NON, ne pratiquant pas d'activités non agricoles. A Luchiga, 10 ménages (33\%) pratiquent des activités non agricoles et 20 ménages (67\%) ne pratiquent pas d’activités non agricoles.

Signalons à cet effet que, nous nous sommes intéressés aux activités non agricoles, mais la majorité des ménages de la collectivité chefferie de Luhwindja pratiquent aussi des activités agricoles, soit 
Impact des activités non agricoles sur la sécurité alimentaire au Sud-Kivu mo...

de manière directe comme activité principale ou indirecte pour certains d'entre eux qui veulent renforcer ou accumuler un revenu pouvant leur permettre de faire face à l'insécurité alimentaire et répondre à d'autres besoins de nécessité de la famille.

La population cible de cette étude est donc constituée par les ménages ruraux de la chefferie de Luhwindja qui pratiquent des activités non agricoles et agricoles.

L'enquête proprement dite a été réalisée en juillet 2016. La taille de l'échantillon a été obtenue en partant de la formule des proportions (38) qui est la suivante :

$$
n_{\mathrm{o}}=\frac{Z_{1-\alpha / 2}^{2} \cdot P \cdot(1-p)}{d_{\mathrm{o}}^{2}}
$$

Avec :

$$
Z_{1-\alpha / 2}^{2}
$$

la valeur critique qui est de 1,96 au niveau de confiance de 95\%

$\mathrm{p}$ : proportion des ménages qui pratiquent des activités non agricoles

$1-\mathrm{p}=\mathrm{q}:$ la proportion des ménages qui ne pratiquent pas d'activités non agricoles

do : marge d'erreur

Se référant à ce qui précède, nous calculons les tailles d'échantillon pour chaque groupement, ce qui nous donne :

$$
\begin{aligned}
& n_{0} \text { Bujiri }=\frac{(1,96)^{2}(0,233)(0,766)}{(0,1)^{2}}=68,56 \\
& n_{0} \text { Luchiga }=\frac{(1,96)^{2}(0,33)(0,66)}{(0,1)^{2}}=83,67
\end{aligned}
$$

Étant donné que la taille d'échantillon dans chaque groupement est connue, il sied de trouver la 
taille d'échantillon corrigée dans chaque site choisi et ceci en se servant de la formule suivante :

$$
n_{c}=\frac{n_{0} x N}{N+n_{0}} \text { ou } n_{c}=\frac{n_{0}}{1+\frac{\left(n_{0}-1\right)}{N}}
$$

$\mathrm{N}$ étant le total de la population dans chaque groupement, ce qui ressort du tableau suivant :

\begin{tabular}{|l|l|l|l|l|l|l|}
\hline$N^{\circ}$ & GROUPEMENT & HOMMES & FEMMES & GARÇONS & FILLES & Total Groupement \\
\hline 1 & BUJIRI & 1622 & 1953 & 2454 & 2632 & 8661 \\
\hline 2 & LUCHIGA & 4591 & 4933 & 5992 & 6121 & 21637 \\
\hline
\end{tabular}

Source : Rapport annuel de la collectivité chefferie, 2015

Cependant, de cette formule, nous allons calculer la taille d'échantillon pour chaque groupement et leur somme permettra de trouver la taille d'échantillon total pour toute la population de notre zone d'étude.

$$
n_{1_{c}}=\frac{68,56}{1+\frac{(68,56-1)}{8661}}=\frac{68,56}{1,0078004}=68,029 \approx 68 \text { est la taille de l'échantillon à Bujiri }
$$

$$
n_{2_{C}}=\frac{83,67}{1+\frac{(83,67-1)}{21637}}=\frac{83,67}{1,00382}=83,351 \approx 83 \text { est la taille de l'échantillo } n \text { à Luchiga }
$$

Ainsi, la taille totale d'échantillon sera :

$$
\begin{aligned}
& n_{C}=n_{1 C}+n_{2 C} \Rightarrow n_{C}=68+83 \\
& \Rightarrow n_{C}=151 \text { Personnes } \quad \text { Avec } n_{C}=n \text { corrigée }
\end{aligned}
$$

Se référant à ce qui précède (sur base de la pré-enquête réalisée) et sur base des caractéristiques de notre échantillon qui était respectivement de 23\% pour Bujiri et 33\% pour Luciga, cela donne les tailles d'échantillons respectives qui suivent : 


$$
n_{0} \text { Bujiri }=\frac{(1,96)^{2}(0,233)(0,766)}{(0,1)^{2}}=68 \text { et } n_{0} \text { Luchiga }=\frac{(1,96)^{2}(0,33)(0,66)}{(0,1)^{2}}=83
$$

Par ailleurs, la taille totale d'échantillon ayant fait l'objet de cette étude est $: n=n_{1}+n_{2}$;

ainsi $n=68+83=151$ ménages.

Le score de consommation alimentaire (SCA) 1 utilisé généralement par le PAM a été obtenu par la collecte et l'analyse des scores de diversité alimentaire et de fréquence de la consommation alimentaire. Les seuils obtenus ont alors été appliqués aux scores pour déterminer les modèles de consommation alimentaire "pauvre (faible)", "limite" et "acceptable". Les scores des ménages furent ensuite comparés aux seuils préétablis qui indiquent la situation de la consommation alimentaire des ménages. En outre, l’indice de stratégie de survie 2 a été également calculé.

\section{Analyses statistiques}

Les données ont été collectées et soumises à une analyse multidimensionnelle à l'aide de la programmation $\mathrm{R}$ et SPSS. La sécurité alimentaire a été analysée à l'aide du modèle LOGIT binaire sur base d'une variable comprenant deux modalités et encodées sous une forme binaire à savoir : « la sécurité alimentaire »(1) ou « insécurité alimentaire »(0).

La fonction suivante incorpore les variables explicatives $\underline{3}$ ( ) de la sécurité alimentaire et détermine l’impact des activités non agricoles sur la sécurité alimentaire des ménages.

\section{Résultats}

\section{Description des données}

Le tableau ci-dessous présente les caractéristiques de notre échantillon. 
Tropicultura Tropicultura 2295-8010 Volume 39 (2021) Numéro 2, 1761

\section{Tableau 1. Résultats descriptifs}


Impact des activités non agricoles sur la sécurité alimentaire au Sud-Kivu mo...

\begin{tabular}{|c|c|c|c|c|c|c|}
\hline \multirow[b]{2}{*}{ Caractéristiques socio-économiques } & & \multicolumn{5}{|l|}{ Variables } \\
\hline & & Moyenne & $\begin{array}{l}\text { Ecart- } \\
\text { type }\end{array}$ & $\mathrm{CV}$ & Min & Max \\
\hline \multicolumn{2}{|l|}{ Age de la personne interrogée } & 42,6 & 12,2 & 0,286 & 25 & 60 \\
\hline \multicolumn{2}{|l|}{ Taille de ménage } & 8 & 2,4 & 0,289 & 4 & 14 \\
\hline \multicolumn{7}{|l|}{ Fréquences par Groupements } \\
\hline \multicolumn{2}{|l|}{ Caractéristiques de l'échantillon } & Effectifs & $\%$ & Bujiri & Luciga & Total \\
\hline \multirow[t]{2}{*}{ Sexe des enquêtés } & $\mathrm{F}$ & 82 & 54 & 37 & 45 & \multirow[t]{2}{*}{151} \\
\hline & M & 69 & 46 & 31 & 38 & \\
\hline \multirow[t]{4}{*}{ Situation matrimoniale } & Célibataire & 20 & 13 & 10 & 10 & \multirow[t]{4}{*}{151} \\
\hline & Divorcé & 3 & 2 & 3 & 0 & \\
\hline & Marié & 126 & 83 & 54 & 72 & \\
\hline & Veuf (veuve) & 2 & 1 & 1 & 1 & \\
\hline \multirow[t]{4}{*}{ Niveau d'éducation } & Analphabète & 23 & 15 & 12 & 11 & \multirow[t]{4}{*}{151} \\
\hline & Primaire & 22 & 15 & 11 & 11 & \\
\hline & Secondaire & 87 & 58 & 36 & 51 & \\
\hline & Sup/ Université & 19 & 13 & 9 & 10 & \\
\hline \multirow[t]{2}{*}{ Activité principale du chef de ménage } & Activité agricole & 103 & 68 & 45 & 58 & \multirow[t]{2}{*}{151} \\
\hline & $\begin{array}{l}\text { Activité non } \\
\text { agricole }\end{array}$ & 48 & 32 & 23 & 25 & \\
\hline \multirow{5}{*}{$\begin{array}{l}\text { Activité non agricole exercée par le chef de } \\
\text { ménage }\end{array}$} & Artisanat & 14 & 9 & 7 & 7 & \multirow[t]{5}{*}{151} \\
\hline & Commerce & 88 & 58 & 40 & 48 & \\
\hline & Coupe couture & 17 & 11 & 9 & 8 & \\
\hline & Enseignement & 17 & 11 & 5 & 12 & \\
\hline & Fonctionnaire & 15 & 10 & 7 & 8 & \\
\hline \multicolumn{2}{|l|}{ Total des ménages enquêtés } & 151 & & & & \\
\hline
\end{tabular}


Source : Nos analyses par SPSS confectionnées en Excel

Sur base des données officielles de la collectivité-chefferie de Luhwinja, notamment la population totale des groupements, l'effectif des femmes représente $51,9 \%$ de la population totale contre $48,1 \%$ pour celui des hommes (27). Il ressort de ce tableau que la majorité de nos enquêtés sont des personnes du sexe féminin dans les deux groupements, soit $54 \%$ de l'ensemble des personnes interrogées. Cela s'explique par le fait que ce sont les femmes qui sont les plus impliquées dans les domaines touchant à l'alimentation familiale et s'intéressent davantage à la situation alimentaire des ménages.

Soixante-neuf hommes dans l'ensemble de l'échantillon, dont 31 dans le groupement de Bujiri et 38 dans le groupement de Luciga, soit $46 \%$, ont été enquêtés dans les deux groupements considérés de la collectivité-chefferie de Luhwinja. Notre échantillon montre que les données statistiques sont conformes et représentatives de la population paysanne de la collectivité-chefferie de Luhwinja.

On observe que dans la collectivité-chefferie de Luhwinja, l'activité agricole est exercée comme activité principale pour $68 \%$ de l'échantillon tandis que l'activité non agricole concerne $32 \%$ de l'échantillon. Cela résulte du fait que généralement, dans les milieux ruraux, en termes de temps d'occupation par les ménages, l'agriculture est considérée comme une activité principale (pour 46 enquêtés sur 68 à Bujiri et 58 enquêtés sur 83 à Luciga) et les activités non agricoles sont considérées comme ne faisant pas partie du vécu quotidien pour certains ménages des milieux ruraux ; ce qui les classe parmi les activités secondaires.

Mais le pourcentage des ménages qui considèrent les activités non agricoles comme activité principale dans la collectivité-chefferie de Luhwinja (23 enquêtés sur 68 à Bujiri et 25 enquêtés sur 83 à Luciga) n'est pas négligeable et s'explique par le fait que, dans ces milieux, le revenu agricole est devenu de plus en plus faible pour assurer la satisfaction des besoins des ménages agricoles.

De ce fait, ces ménages se concentrent beaucoup plus sur les activités extra-agricoles $\underline{4}$ vu leur part dans le revenu du ménage bien que la pratique des activités liées à l'agriculture reste primordiale et n'a pas été abandonnée par les paysans des deux groupements considérés. Signalons aussi, qu'après analyse des données, les activités non agricoles exercées dans les deux groupements de la collectivité-chefferie de Luhwinja se rapportaient au petit commerce (58\%), à l'enseignement et la coupe-couture (11\% chacune), au métier de fonctionnaire (10\%) et à l'artisanat (9\%).

Parmi ces activités, le commerce est l'activité la plus fréquemment pratiquée par le chef de ménage (soit 40 enquêtés sur 68 à Bujiri et 48 enquêtés sur 83 à Luciga). Ensuite, on trouve l'enseignement et la coupe-couture et enfin l'artisanat, plus particulièrement l'exploitation artisanale de l'or, fortement répandue dans le milieu rural étant donné les gisements et l'exploitation mis en valeur par certaines organisations non gouvernementales.

Le tableau 1 ci-dessus montre que diverses activités non agricoles sont exercées par les ménages. Une cause probablement majeure est que la faiblesse de leurs revenus agricoles, les pousse à exercer des activités extra-agricoles afin d'améliorer le niveau de leur revenu.

La pratique d'activités non agricoles exige généralement certaines compétences ; cela se traduit par le fait que la majorité des personnes enquêtées possède un niveau d'études secondaires (58 \%) et sont disposées à obtenir un diplôme, un brevet ou un autre niveau d'études leur permettant 
Impact des activités non agricoles sur la sécurité alimentaire au Sud-Kivu mo...

d'évoluer dans la pratique d'activités extra-agricoles. Les paysans ayant fait des études supérieures ont acquis les compétences nécessaires leur permettant d'exercer certaines activités non agricoles dans le but de diversifier leurs sources de revenu.

\section{Raisons de la diversification des activités}

L'agriculture joue un rôle moteur dans le développement. Elle intervient dans la création des revenus et a un rôle primordial pour la sécurité alimentaire des ménages (2). Elle fournit non seulement les aliments pour l'autoconsommation et la vente, mais elle soutient de nombreux ménages et communautés dans les zones tant rurales qu'urbaines et contribue ainsi à la réduction de la pauvreté $(20,36)$.

Selon les enquêtes menées dans les deux groupements de la collectivité-chefferie de Luhwinja sur la question principale de la diversification des activités, les enquêtés diversifient leur activité dans le but d'augmenter leur revenu vu que la production agricole est insuffisante au cours de la période considérée et que le niveau du revenu agricole est jugé très faible.

Ainsi, il convient de préciser que, sur 151 enquêtés dans les deux groupements de la collectivitéchefferie de Luhwinja, $49 \%$ ont un revenu lié à la diversification des activités (Soit $46 \%$ dans le groupement de Bujiri et $52 \%$ dans le groupement de Luciga). Ces derniers affectent ce revenu non pour acheter des aliments et ainsi assurer leur sécurité alimentaire, mais bien pour répondre à d'autres besoins et dépenses du ménage, entre autres le logement, la scolarisation, les soins de santé, etc. Le revenu tiré des activités extra-agricoles étant en grande partie utilisé pour d'autres dépenses, on constate donc que l'alimentation n'est pas une priorité, étant donné que l'agriculture pratiquée est de subsistance, liée à l'autoconsommation et génère de faibles revenus parfois insuffisants pour couvrir les besoins alimentaires complémentaires. Il en résulte qu'une affectation du revenu extra agricole à des fins essentiellement non alimentaires peut conduire à l'insécurité alimentaire.

Tel que le montre le graphique ci-dessous, les ménages de deux groupements de la collectivitéchefferie de Luhwinja diversifient leurs activités pour plusieurs raisons avancées qui sont, par ordre de fréquence décroissante, le renforcement de leur revenu, une production agricole insuffisante sur leur exploitation, la satisfaction des besoins familiaux non alimentaires (logement, scolarisation, soins de santé), et la couverture de la période de soudure suite à la production agricole insuffisante. Ainsi donc, une mauvaise affectation des ressources est observée vu qu'une part importante de ce revenu supplémentaire issu des activités non agricoles est affectée non pas à l'alimentation mais à la satisfaction d'autres besoins familiaux. 


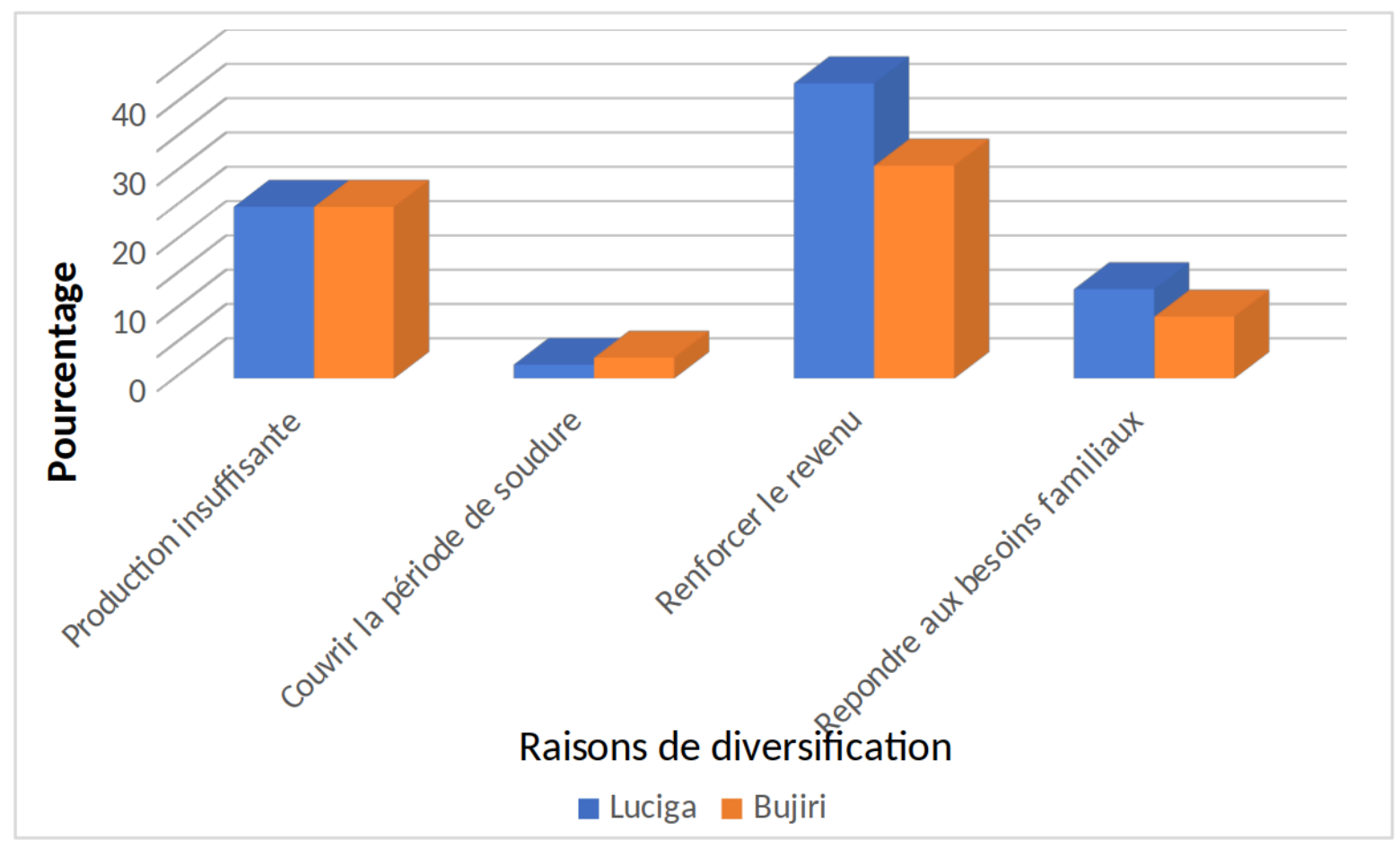

Graphique $\mathbf{N}^{\circ} 1$ : Raisons de la diversification des activités

\section{Analyse comparative des parts des activités non agricoles (ANA) et des activités agricoles (AA)}

Le revenu monétaire tiré des activités non agricoles dans le revenu global du ménage est de 138 \$ par mois avec un écart type de 70 \$. L'autoconsommation n'étant pas estimée en valeur, la part des activités agricoles s'élève à $23 \$$ par mois. Cela étant, la part commercialisée est faible pour satisfaire les besoins des ménages, ce qui fait que les ménages se concentrent plus sur les activités extra-agricoles afin d'accroître leur revenu. Ce qui traduit dès lors la part importante du revenu non agricole dans le revenu total du ménage.

Remarquons aussi, comme signalé précédemment que, les autres dépenses telles que le financement des activités agricoles et l'épargne du ménage proviennent du revenu tiré des activités extraagricoles. De même, pour le financement des autres dépenses, la part des activités non agricoles est toujours supérieure à celle des activités agricoles. Nous observons que la dispersion autour de la moyenne pour toutes ces variables est grande. Il en résulte qu'un ménage qui atteint un niveau de revenu de 250 \$ appartient pourtant au même groupement qu'un autre ménage qui ne dispose que d'un revenu de 40 \$, ce qui démontre des inégalités entre les ménages au sein de la collectivité-chefferie de Luhwinja.

Nous constatons alors qu'en comparant les deux sources de revenu, sans valoriser l'autoconsommation, les dépenses familiales sont financées par les activités extra-agricoles et les activités agricoles ne comblent qu'en partie les dépenses alimentaires. Il y a donc une différence significative entre la diversification et à la non-diversification des activités par les paysans de la collectivité-chefferie de Luhwinja. 
Impact des activités non agricoles sur la sécurité alimentaire au Sud-Kivu mo...

\section{Analyse de la sécurité alimentaire}

Partant du modèle évoqué plus haut et des variables expliquant l'état de la sécurité alimentaire dans la collectivité-chefferie de Luhwinja, après analyse, le résultat de la régression a montré que le stock alimentaire issu des activités agricoles a une influence significative sur la sécurité alimentaire des ménages. Le nombre de champs ainsi que la superficie détenue par un ménage rural sont corrélés positivement avec le niveau de la production agricole et de quantité auto-consommée, affectant positivement l'état de sécurité alimentaire des ménages. Dans la mesure où les ménages exerceraient une activité non agricole, celle-ci permettrait de satisfaire les besoins familiaux de base, non seulement en termes d'alimentation, mais aussi en termes d'autres besoins tels que la scolarisation, le logement, etc.

Ainsi, les résultats de la régression ont montré que seulement 4 variables du modèle étaient significatives au seuil de 5\%, à savoir l'âge, le niveau d'études, le stock provenant des activités non agricoles, et les dépenses alimentaires financées par les activités non agricoles. Ces variables affectent significativement au seuil de $5 \%$ la situation de la sécurité alimentaire des ménages de la collectivité-chefferie de Luhwinja.

Quant au score de consommation alimentaire $\underline{5}$ dans les deux groupements considérés, la sécurité alimentaire des ménages a été évaluée en procédant à la combinaison des analyses de la consommation alimentaire et de l'accès économique à la nourriture $\underline{6}$. Les enquêtes effectuées dans la collectivité-chefferie de Luhwinja montrent que la majorité des enquêtés (72\%) se trouve dans la catégorie pauvre pour laquelle l'alimentation n'est pas adéquate au besoin alimentaire, c'est-à-dire que les ménages consomment en quantité sans consommer en qualité.

Seulement une minorité de nos enquêtés à un accès facile au lait et aux produits animaux (poisson, viande), pourtant aliments à coefficient de pondération élevé et fixé par le PAM à 4. Ces aliments sont riches en protéines de bonne qualité, en micronutriments, nutritifs, facilement absorbables, denses en énergie et riche en gras.

Ce score de consommation alimentaire est évalué selon que les ménages diversifient ou pas leurs activités, étant donné que les aliments de base dans la collectivité-chefferie de Luhwinja sont en premier lieu des tubercules et des céréales, principalement du manioc et du maïs, avec une fréquence de consommation hebdomadaire moyenne de 5 fois sur les 7 jours de la semaine. Suivent ensuite les légumes, les feuilles et les légumineuses (haricots) consommés en moyenne trois fois par semaine.

En analysant les différentes stratégies auxquelles font appel ces ménages ruraux pour faire face à l'état d'insécurité alimentaire, signalons que les ménages recourent beaucoup à l'une ou à une autre combinaison des cinq stratégies de survie suivantes : manger des aliments moins appréciés/ moins chers, emprunter de la nourriture ou dépendre de l'aide d'amis ou de parents, limiter les portions et quantités du repas, réduire la consommation des adultes en faveur des enfants, et réduire le nombre des repas pris par jour.

L'indice de stratégie de survie étant un indicateur de substitution de l'accès aux aliments, il révèle la sévérité des stratégies auxquelles les ménages ont recours pour faire face à des déficits dans leur consommation alimentaire. Ces stratégies peuvent affecter négativement le niveau de sécurité alimentaire des ménages, car le recours à celles-ci fait que le ménage risque de ne plus pouvoir remplir les conditions nécessaires pour une nutrition adéquate. Ces ménages seront donc en train 
de manger pour manger mais non de manger comme il faut. Le recours fréquent des ménages à ces stratégies de survie affecte donc négativement le niveau de sécurité alimentaire des ménages de la collectivité-chefferie de Luhwinja.

\section{Discussion}

Les résultats de notre étude décrivent la contribution des activités extra-agricoles au revenu des ménages dans la collectivité-chefferie de Luhwinja et l'importance de la diversification des activités par les ménages. D'autres études sur la diversification des activités ont été menées sur les Hautes terres. Pour IFPRI et FOFIFA (21), la diversification apporte une contribution au niveau du revenu des ménages estimée à hauteur de 25 à $40 \%$, surtout quand il s'agit de la part des activités non agricoles. Ce qui rejoint nos résultats selon lesquels une part importante du revenu des ménages de la collectivité-chefferie de Luhwinja provient des activités extra-agricoles.

Cette étude démontre la mauvaise gestion du revenu issu des activités extra-agricoles par les ménages de deux groupements de la collectivité-chefferie de Luhwinja étant donné que la grande partie de celui-ci couvre les autres dépenses familiales en dehors des besoins alimentaires qui ne sont comblés que par les activités agricoles, bien que le revenu et la production soient faibles.

Hazell et Haggblade (20) ont trouvé, lors de leurs investigations que les ménages agricoles, pour faire face à l'insécurité alimentaire, se consacrent de façon quasi exclusive aux activités agricoles et n'entreprennent que de façon marginale des activités rurales non agricoles. Cela résulte du fait qu'au Sud-Kivu, en amont, les sols sont très dégradés et ne permettent pas aux ménages de produire suffisamment afin de satisfaire les besoins et les charges du ménage.

En aval, la forte explosion démographique, corrélée à une absence de politique foncière conséquente dans la distribution des terres arables, conduit à cultiver sur des superficies très réduites ne pouvant pas permettre la pratique d'une agriculture tant intensive qu'extensive. Par contre, nos résultats corroborent ceux de Nong Zhu (31) qui a démontré que la participation aux activités non agricoles est capitale pour le ménage et pour le développement rural. En effet, les ménages en situation de sécurité alimentaire, dans notre cas, sont ceux qui ont su répartir le revenu issu de l’activité non agricole dans les dépenses alimentaires.

Bakary K. (4) et Kidane et al. (23), s'intéressant uniquement aux activités informelles non agricoles, montrent que ces dernières contribuent positivement à la sécurité alimentaire des ménages. Ils estiment que cela est dû à l'élaboration et la mise en place des politiques et stratégies relatives aux activités informelles non agricoles, leurs résultats corroborant ainsi les nôtres. Par ailleurs, selon le rapport SOS Faim (2010) dans son dossier numéro 97, les revenus non agricoles des ménages ruraux congolais représentent 20 à $30 \%$ des ressources familiales.

Analysant systématiquement l'impact des activités non agricoles sur la pauvreté et l'inégalité rurales dans les groupements de Bugorhe et d'Irhambi-Katana au Sud-Kivu, dans l'objectif de montrer que la diversification des activités a un effet positif sur la vie socio-économique du ménage, Furaha et al. (18) ont démontré qu'un ménage ayant diversifié ses sources de revenu échappe aux chocs négatifs et voit ses revenus s'améliorer, entrainant une réduction les inégalités entre les ménages. Ce qui confirme nos résultats car au Sud-Kivu montagneux, dans les groupements de Bujiri et Luciga, la diversification des activités économiques par un ménage agricole a un effet positif sur le niveau de vie des ménages à travers l'amélioration du revenu et de la sécurité alimentaire de ces ménages, si 
Impact des activités non agricoles sur la sécurité alimentaire au Sud-Kivu mo...

et seulement si la bonne gestion de ce revenu issu des activités extra-agricoles est assurée.

Par ailleurs, la plupart des recherches soulignent que la distribution du revenu non agricole est plus inégale que celle du revenu agricole $(5,26,32)$, confirmant les résultats obtenus lors de nos investigations.

En outre, un ménage agricole peut avoir la capacité de diversifier les activités économiques, mais ne pas être en mesure d'assurer la bonne gestion de ces dernières. C'est ce qui a été observé dans notre étude au niveau de la collectivité-chefferie de Luhwinja au Sud-Kivu montagneux, avec un revenu des activités non agricoles qui représente une part importante du revenu total du ménage.

\section{Conclusion}

Le secteur rural non agricole occupe une place importante dans les économies des ménages ruraux en raison de son influence sur la production constituant ainsi une source des services rémunérateurs et contribuant indirectement à l'achat des produits alimentaires et d'autres ingrédients clés du dynamisme de l'agriculture dans les ménages agricoles.

Dans l'objectif d'analyser l'impact de l'activité non agricole sur la sécurité alimentaire des ménages de la province du Sud-Kivu, et plus précisément dans la collectivité-chefferie de Luhwinja, les activités extra-agricoles occupent une place importante visant à satisfaire les besoins divers des ménages. Ainsi, la diversification des activités a des effets positifs sur la vie socio-économique des ménages, ces derniers recourent à celle-ci pour générer un revenu capable de satisfaire en moyenne leurs besoins familiaux que la production agricole ne parvient pas à satisfaire.

Il existe cependant une relation significative entre les dépenses financées par les activités agricoles et celles financées par les activités non agricoles et on observe à cet effet une complémentarité vu qu'excepté l'autoconsommation, les activités agricoles financent les besoins alimentaires des ménages et la part du revenu issu des activités non agricoles subvient à d'autres besoins du ménage. Ainsi donc, si la bonne gestion de ce revenu extra-agricole était assurée, et une part importante destinée à la consommation alimentaire, les paysans de deux groupements de la collectivité-chefferie de Luhwinja seraient bel et bien en sécurité alimentaire.

Dans l'avenir, les contributions de ces activités et la bonne gestion des revenus issus des activités extra-agricoles revêtiront une importance croissante pour le développement durable et la sécurité alimentaire des ménages ruraux au Sud-Kivu montagneux en République démocratique du Congo.

\section{Bibliographie}

1. Adams R.H.J., 1994. Non-farm income and inequality in rural Pakistan: Decomposition analysis. The Journal of Development Studies, $\underline{31}(1), 110-133$.

2. Andrianirina N., 2013. L'agriculture pour le développement : pertinence et limites à l'échelle des ménages ruraux. Une approche dynamique comparative pour trois régions de Madagascar. Thèse de doctorat : Centre international d'études supérieures en sciences agronomiques - SupAgro, Montpellier, France, 170 p.

3. Aruna Sefu et al., 2020. Climate risks assessment, opportunities related to the evolution of ecosystems and limitation of the provion of ecosystem services : Lanscape case of 
South. Kivu (Albertine riflt,DRC). International Journal of Research -Granthaalaya. 2020, 8 (7), 98-117.

4. Bakary K., 1992. Les activités informelles non agricoles et sécurité alimentaire au Sahel. Document de travail 92-01, INSAH, Bamako.

5. Barham B. \& Boucher S., 1998. Migration, remittances, and inequality: estimating the net effects of migration on income distribution. Journal of Development Economics, 55, 307-331.

6. Barreta C.B., Reardon T. \& Webb P., 2001. Nonfarm income diversification and household livelihood strategies in rural Africa: concepts, dynamics, and policy implications. Food Policy, 26, 315-331.

7. Casinga M.-C., 2018. Cartographie de la collectivité-chefferie de Luhwinja, Province du Sud-Kivu, République démocratique du Congo.

8. Casinga M.-C., Cirimwami L., Amzati G. \& Lubobo A., 2016. Effet du stress hydrique sur le criblage variétal des haricots communs (Phaseolus vulgaris) dans le marais du SudKivu montagneux. Afrique Science, 12(1), 335-344.

9. Casinga C.M., Neema C.A., Kajibwami C.M., Nabahungu N.L. \& Mambani B.P. (2017). Effect of Soil Moisture Regimes on Seed Iron and Zinc Concentration of Biofortified Bean Genotypes against Malnutrition in Sud-Kivu Highlands. Journal of Agricultural Science ; Vol. 9, No. $12 ; 241-252$. DOI : 10.5539/jas. v9n12p241

10. Crawford Jr., Singh U. \& Breman,H. Résoudre les problèmes relatifs à l'acidité des sols dans la Région des Grands Lacs de l'Afrique Centrale. Un rapport du projet CATALIST. IFDC. 2008, $132 \mathrm{p}$.

11. De Saint Moulin L. \& Kalombo J.-L., 2005. Atlas de l'organisation administrative de la République démocratique du Congo (Fonds de plan de l'Institut géographique du Congo), Centre d'Etudes pour l'Action Sociale (CEPAS), Kinshasa, p. 132.

12. DSRP (2005), Analyse participative de la pauvreté en RDC, Rapport national, Ministère de Plan, RDC

13. Escobal J., 2001. The determinants of nonfarm income diversification in rural Peru. World Development, $\underline{29}(3), 497-508$.

14. FAO \& PAM., 2013. République de Côte d'Ivoire : enquête sur l'insécurité alimentaire et la vulnérabilité à l'Ouest et au Nord-Ouest, FAO, Rome.

15. FAO, 1998. The state of food and agriculture 1998, FAO, Rome, $371 \mathrm{p}$.

16. FAO, 2014. L'état de l'insécurité alimentaire dans le monde. Objectifs internationaux de réduction de la faim : des progrès inégaux, FAO, Rome, $61 \mathrm{p}$.

17. Faure M.-E., 2015. Evaluation de la sécurité alimentaire en situation d'urgence : Guinée Conakry. World Food Programme, Rome, 29 p. 
Impact des activités non agricoles sur la sécurité alimentaire au Sud-Kivu mo...

18. Furaha G., Mastaki L. \& Lebailly Ph., 2013. L'impact des activités non-agricoles sur la pauvreté et l'inégalité rurales. Cas des groupements Bugorhe et Irhambi-Katana (territoire de Kabare, province du Sud-Kivu). In : Société française d'Economie rurale, 7 èmes Journées de Recherches en Sciences Sociales : actes du colloque, Angers, France, 12-13 décembre 2013, SFER, Angers, 26 p.

19. Goh C-C., Xubei L. \& Nong Z., 2009. Income growth, inequality and poverty reduction: a case study of eight provinces in China. China Economic Review, 20(3), 485-496.

20. Hazell P., Poulton C., Wiggins S. \& Dorward A., 2007. The future of small farms for poverty reduction and growth. 2020 Discussion Paper $N^{\circ}$ 42, IFPRI, Washington, D.C, 38 p.

21. IFPRI \& FOFIFA, 1998. Determinants of income generation and welfare of rural households in Madagascar and implications for policy. USAID, Washington, D.C., 337 p.

22. Kasongo L.M., 2008. Système d'évaluation des terres à multiples échelles pour la détermination de l'impact de la gestion agricole sur la sécurité alimentaire au Katanga, $R D$ Congo. Thèse de doctorat : Université de Gand, Gand, Belgique, 309 p.

23. Kidane W., Maetz M. \& Dardel Ph., 2003. Sécurité alimentaire et développement agricole en Afrique subsaharienne : dossier pour l'accroissement des soutiens publics. Rapport principal, FAO, Rome, $108 \mathrm{p}$.

24. Lebailly Ph., 2010. Rapide évaluation de l'impact de la crise du secteur minier de la zone Lubumbashi-Kolwezi de la province de Katanga (RD Congo) et des potentialités en termes de promotion et de l'emploi : la problématique agricole, document élaboré pour le Bureau international du travail, BIT, Genève, $34 \mathrm{p}$.

25. Lebailly P., Michel B., Ntoto R. (2014), Quel développement agricole pour la RDC? In: Tshonda, J.O., editor. Conjonctures Congolaises

26. Leones P. \& Feldman S., 1998. Nonfarm activity and rural household income: Evidence from Philippine microdata. Economic Development and Cultural Change, 46(4), 789-806.

27. Ministère de l’Agriculture, 2015. Rapport annuel de la collectivité chefferie de Luhwinja, Province du Sud-Kivu, RDC.

28. MINISTERE DU PLAN, 2006, Unité de Pilotage du Processus d’Elaboration et de Mise en Euvre de la Stratégie pour la Réduction de la Pauvreté, Rapport National, DSRP, RDC, Mars 2006

29. Monographie du Sud-Kivu, 2005. Unité de pilotage du Processus DSRP, Kinshasa/Gombe.

30. Ndiaye M., 2014. Indicateurs de la sécurité alimentaire, Programme Alimentaire Mondial, Rome, p. 27.

31. Nong Z., 2002. Déterminants de la participation aux activités non agricoles et du revenu des ménages ruraux : le cas de la Chine. Document de travail EC 2002.23, Centre d'Etudes et de Recherches sur le Développement International (CERDI), Clermont Ferrand, $19 \mathrm{p}$. 
32. Reardon T. \& Taylor J.E., 1996. Agroclimatic shock, income inequality, and poverty, evidence from Burkina Faso. World Development, 24(5), 901-914.

33. SOS Faim, 2010. Activités non agricoles et territoires ruraux. Défis Sud $n^{\circ}$ 97, rapport bimestriel, SOS Faim, Bruxelles.

34. Walder A.G., 2002. Income determination and market opportunity in rural China, 1978-1996. Journal of Comparative Economics, 30(2), 354-375.

35. WFP, 2011. Résultats de l'enquête approfondie sur la sécurité alimentaire des ménages en situation d'urgence en Province Orientale (EFSA). Rapport final, RDC

36. World Bank, 2007. World Development Report 2008: Agriculture for development. The World Bank, Washington, D.C., 365 p.

37. Yang D.T., 2004. Education and allocative efficiency: household income growth during rural reforms in China. Journal of Development Economics, 74(1), 137-162.

38. https://fr.surveymonkey.com/mp/sample-size-calculator/ consulté le 09/02/21

\section{Notes}

1 Le Score de Consommation Alimentaire est un indicateur qui permet de déterminer la situation de sécurité alimentaire des ménages sur base de la valeur nutritionnelle des aliments consommés dans les ménages enquêtés au cours d'une semaine en tenant compte des coefficients de pondération définis par le PAM pour 8 catégories de produits : 2 pour les céréales et tubercules, 3 pour les légumineuses, 1 pour les légumes et les fruits, 4 pour la viande et le poisson, 0,5 pour le sucre et l'huile et 4 pour les produits laitiers. Cet indicateur permet de classifier la situation alimentaire des ménages en trois catégories, notamment pauvre (SCA $<21)$, limite (SCA entre $21,5-35)$ et acceptable $(\mathrm{SCA}>35)(14,30)$.

$\underline{2}$ L'indice de stratégie de survie est un indicateur permettant de déterminer le niveau de vulnérabilité des ménages mais aussi le niveau de risque menaçant les moyens de subsistance des ménages. Il tient compte des différentes stratégies ainsi que la fréquence et la sévérité de celles-

ci. ISS = stratégie 1 (fréquence $\times$ sévérité) + stratégie 2 (fréquence $\times$ sévérité) + stratégie 3 (fréquence $\times$ sévérité) + ... (14). Il s'agit d'un indicateur de substitution de l'accès aux aliments révélant la sévérité des stratégies auxquelles les ménages ont recours pour faire face à des déficits dans leur consommation alimentaire.

3 Ces variables explicatives de la SA étant l'âge, le niveau d'étude, la taille du ménage, aliments en stock, part de l'activité agricole et non agricole dans le revenu du ménage, le nombre de repas par jour, les dépenses alimentaires financées par les activités agricoles et non agricoles et le nombre des repas par jour.

4 Ce sont des activités exercées en dehors de l'agriculture et le revenu issu de ces activités est qualifié dans le cas de cette étude, de revenu non agricole

$\underline{5}$ Le Score de Consommation Alimentaire est un indicateur qui permet de déterminer la situation de sécurité alimentaire des ménages sur base de la valeur nutritionnelle des aliments consommés dans les ménages enquêtés au cours d'une semaine en tenant compte des coefficients de 
Impact des activités non agricoles sur la sécurité alimentaire au Sud-Kivu mo...

pondération définis par le PAM pour 8 catégories de produits : 2 pour les céréales et tubercules, 3 pour les légumineuses, 1 pour les légumes et les fruits, 4 pour la viande et le poisson, 0,5 pour le sucre et l'huile et 4 pour les produits laitiers. Cet indicateur permet de classifier la situation alimentaire des ménages en trois catégories, notamment pauvre (SCA $<21$ ), limite (SCA entre 21,5-35) et acceptable (SCA>35) $(14,30)$.

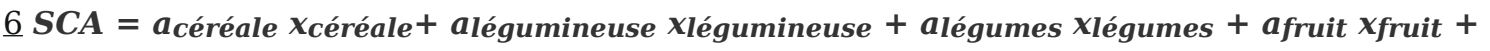

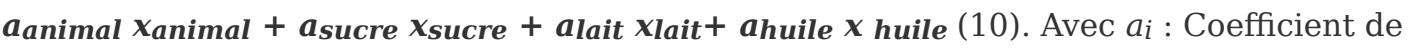
pondération attribué au groupe d'aliments; $x_{i}$ : Fréquence de consommation relative à chaque groupe d'aliments ( $\leq 7$ jours)

PDF généré automatiquement le 2023-04-26 13:20:12

Url de l'article : https://popups.uliege.be/2295-8010/index.php?id=1761 